\title{
Finding the origin of pulmonary emboli with a total-body magnetic resonance direct thrombus imaging technique
}

\author{
Kirsten van Langevelde, ${ }^{1}$ Alexandr Šrámek, ${ }^{2}$ Patrice W.J. Vincken, ${ }^{3}$ Jan-Kees van Rooden, ${ }^{4}$ Frits R. Rosendaal, ${ }^{1,5}$
} and Suzanne C. Cannegieter ${ }^{1}$

${ }^{1}$ Department of Clinical Epidemiology, Leiden University Medical Center, Leiden, the Netherlands; ${ }^{2}$ Department of Radiology, Leiden University Medical Center, Leiden, the Netherlands; ${ }^{3}$ Department of Radiology, Rijnland Hospital, Leiderdorp, the Netherlands; ${ }^{4}$ Department of Radiology, Haga Teaching Hospital, The Hague, the Netherlands, and ${ }^{5}$ Department of Thrombosis and Haemostasis, Leiden University Medical Center, Leiden, the Netherlands

Pulmonary embolism is considered to originate from embolization of a deep-vein thrombosis, resulting in two manifestations of one disease: venous thrombosis. However, in up to $50 \%$ of patients with pulmonary embolism no deep-vein thrombosis is found with ultrasonography. An explanation for this low proportion is currently lacking. Other imaging modalities may increase the yield of detection of deep-vein thrombosis in the calf or in the abdominal region. Alternatively, not all pulmonary emboli may originate from deep-vein thromboses in the extremities. We searched for the origin of pulmonary emboli, by performing total-body magnetic resonance imaging-scans to visualize thrombi. Ninety-nine patients with a first pulmonary embolism confirmed by computed tomography underwent a magnetic resonance direct thrombus imaging-scan, a validated technique using endogenous contrast. Additionally, acquired and genetic risk factors were assessed. No thrombus was found in 55 patients, whereas a thrombus was identified in 44 patients. The commonest thrombus location was the lower leg; 12 patients had isolated calf vein thrombosis and five had isolated superficial vein thrombosis. A peripheral thrombus was found by magnetic resonance imaging in less than half of patients with pulmonary embolism. We propose several hypotheses to explain the absence of thrombi, such as a cardiac thrombus origin or embolization of the whole deep-vein thrombus. The possibility that pulmonary embolism arises de novo in the lungs, due to local inflammation-driven coagulation, needs to be considered.

\section{Introduction}

Since autopsy studies by Virchow in the mid 1800s, pulmonary embolism (PE) has been thought to originate from embolization of a deep-vein thrombosis (DVT), resulting in two clinical manifestations of one disease: venous thrombosis. However, in up to $50 \%$ of patients with PE no DVT is found by ultrasonography or contrast venography studies. ${ }^{1-6}$ There is currently no suitable explanation for this low proportion of detected thrombi.

Compression ultrasonography (CUS) is presently the leading diagnostic modality for the diagnosis of DVT, with a sensitivity of $96 \%$ and specificity of $98 \%$ for symptomatic proximal DVT? A disadvantage of CUS is its reduced accuracy when it comes to thrombi below the knee or pelvic thrombi. Thrombi in the calf veins extend to proximal veins in 20-30\% of cases, and may embolize from the calves or after extension, contributing to the occurrence of $\mathrm{PE} .{ }^{8}$ Calf veins and pelvic veins may be an overlooked source of $\mathrm{PE}$ that could explain the $20-50 \%$ of thrombi missed by CUS imaging. Other imaging modalities may, therefore, lead to a larger DVT yield, either in the abdominal and pelvic region, or in the calf veins. Combined computed tomography (CT) pulmonary angiography and CT venography has been performed to search for abdominal thrombi. By extending the scan from the lungs to the pelvis and legs, Nchimi et al. found $10 \%$ of DVT above the inguinal ligament. ${ }^{9}$

A validated, new and highly sensitive technique for detecting DVT is magnetic resonance direct thrombus imaging (MRDTI). ${ }^{10,11}$ This is a non-invasive technique that does not require gadolinium contrast. Unlike most imaging techniques, which show a thrombus as a filling defect, MRDTI shows the thrombus itself and suppresses background signals. This method is based on the transformation of hemoglobin into methemoglobin when a thrombus is formed. Using a T1 sequence, methemoglobin in the thrombus gives a strong signal that disappears after about 6 months. MRDTI was found to have a sensitivity of $98 \%$ and a specificity of $96 \%$ compared to ultrasonography or venography in 101 patients with symptomatic DVT. ${ }^{10}$ Since physical accessibility of veins plays no role in MRDTI, this method allows visualization of thrombi at all anatomical loci.

As for the anatomical origin of $\mathrm{PE}$, risk factors for the migration of a DVT towards the lungs are not well known and are clearly not uniform. The most prominent differential risk factor that has been found is the factor $\mathrm{V}$ Leiden mutation, which causes activated protein $C$ resistance and thereby increases thrombotic risk. Factor $\mathrm{V}$ Leiden increases the incidence of DVT (odds ratios ranging from 3 to 10$)^{1,12-15}$ more than that of $\mathrm{PE}$ (odds ratios ranging from 1 to 5 ), ${ }^{16-18}$ compared 
to the incidences in non-carriers of the mutation. This differential effect of factor $\mathrm{V}$ Leiden has become known as the factor $\mathrm{V}$ Leiden paradox, and has not been found for other genetic risk factors for venous thrombosis. ${ }^{19,20}$ Some acquired factors also affect the risk of DVT and PE differently, e.g., the use of oral contraceptives is associated with a 4-fold increased risk of PE and an almost 7-fold increased risk of DVT, ${ }^{19,21}$ while chronic obstructive pulmonary disease, asthma and pneumonia mainly increase the risk of PE. ${ }^{22-24}$ It does not, therefore, appear justified to consider DVT and PE as simply manifestations of one disease ("venous thromboembolism").

We set up the Pulmonary Embolism: Development, Localization, And Risk factors (PEDLAR) study to determine where PE originated from, and to assess whether all PE were accompanied by DVT. When DVT was present, we described the anatomical location and the number of affected veins. In order to do this, we performed totalbody magnetic resonance imaging (MRI) to visualize thrombi from calf veins up to subclavian veins. In addition, we studied to what extent risk factors for isolated PE differ from risk factors for PE with concomitant DVT.

\section{Design and Methods}

The enrollment of patients into the PEDLAR study started in November 2008 and closed in February 2011. All patients from the Leiden University Medical Center (Leiden, the Netherlands) and the Rijnland hospital (Leiderdorp, the Netherlands) above 18 years with a first PE confirmed by CT pulmonary angiography were invited to undergo a total-body MRDTI scan within 7 days after diagnosis. Inclusion into the study in the Rijnland hospital started 1 year later, in October 2009. Approval for this study was obtained from the Medical Ethics Committees of both centers. All participants provided written informed consent according to the Helsinki declaration. Patients with PE were selected at the Radiology departments of the two medical centers. The study comprised a single visit of 1 hour to the Leiden University Medical Center where all tests took place.

The presence of a pacemaker was an exclusion criterion for the study. In addition, claustrophobia and high body weight were contraindications to undergoing MRI scanning. No strict cut-off for body weight was applied, as the distribution of body weight was more important than weight itself to fit into the scanner.

A questionnaire on risk factors for venous thrombosis was filled in by all patients. The questionnaire contained questions on family history of venous thrombosis, recent travel, surgery, cancer, and use of the contraceptive pill. As atrial fibrillation may give rise to thrombus formation in the right heart, and could thereby be a possible source of PE, electrocardiograms were reviewed as well as the medical charts. Patients were categorized as "atrial fibrillation positive" when they had a documented history of atrial fibrillation, or when the electrocardiogram recorded at the time of diagnosis of PE showed atrial fibrillation, and negative in all other cases. Blood was drawn for analysis of the factor V Leiden and prothrombin G20210A mutations. These were determined by polymerase chain reactions using a TaqMan assay. A detailed description of these methods has been published previously.25 When it was not possible to draw blood, DNA was collected via a buccal swab.

The total-body scan was performed on a Philips 1.5 Tesla MRI scanner (Philips Medical Systems, Best, the Netherlands), with extended table construction. The MRDTI protocol consisted of a T1-weighted, three-dimensional gradient-echo sequence devel- oped by Moody and colleagues and modified to Philips equipment by Westerbeek et al. ${ }^{11,26}$ The MRI protocol has been described more extensively in a review on MRI of venous thrombosis. ${ }^{27}$ Pulmonary structures (including the pulmonary arteries) could not be imaged with the MRDTI scan we used, because of the freebreathing scanning protocol. We did not, therefore, attempt to confirm the pulmonary emboli detected by CT pulmonary angiography by MRI.

We aimed to enroll 100 patients with a first PE in this observational study. We assessed the anatomic location of the DVT, the number of affected veins per patient, and whether the affected veins were proximal or distal. Calf vein thrombosis was included in the analysis, and superficial vein thrombosis was documented separately from DVT. Superficial vein thrombosis was often seen in tortuous varicose veins, and thrombus length was not, therefore, measurable. To achieve a clear overall interpretation of the localization of thrombi on MRI, we subdivided the relevant veins into seven segments, which were independent of the side of the thrombosis. The first segment was abdominal, and included the inferior vena cava and the pelvic veins or any other abdominal veins. The second segment was the upper leg, including the common femoral vein, the superficial femoral vein, the profunda femoris vein, and the popliteal vein. The third segment included the calf veins and, as mentioned previously, deep and superficial veins were assessed separately. Occlusion in these three segments could be present in isolation or in combination, i.e., a combination of the abdominal segment and the upper leg, abdominal veins and calf veins, all leg veins (upper and lower leg), and finally the abdominal, upper and lower leg veins together. The magnetic resonance images were scored by consensus of two radiologists (AŠ and CJvR) who both had more than 3 years of experience with magnetic resonance angiography. During the scoring process both radiologists were blinded to the DVT status according to CUS. The prevalence of acquired and genetic risk factors in PE patients with and without concomitant DVT was calculated in percentages, with a corresponding $95 \%$ confidence interval (CI).

\section{Results}

From November 2008 to February 2011 we enrolled 102 PE patients into the study. Figure 1 shows the flowchart of the study by medical center. The majority of participants $(\mathrm{n}=80,78 \%)$ came from the Leiden University Medical Center, whereas $22(22 \%)$ came from the Rijnland hospital. Participation in the Leiden University Medical Center was $40 \%$ and in the Rijnland hospital $31 \%$. All MRI scans took place at the Leiden University Medical Center. Of the 102 patients who were enrolled, 99 successfully completed the MRI. MRI was not completed in three cases due to claustrophobia, a waist circumference too large to fit in the scanner, and a technical problem. The patients' characteristics are shown in Table 1 . The median age of the patients was 54 (range, 18-84) years. There were more male than female participants in the study, $61 \%$ versus $39 \%$, respectively. Of the study participants, 13 had undergone an ultrasound examination of the legs on clinical request. Of these, 10 patients were found to have concomitant DVT.

Table 2 shows the localization of thrombi in all $\mathrm{PE}$ patients. No thrombus was found in $55(56 \%)$ patients, whereas a thrombus was located in $44(44 \%)$ patients. We found no thrombi in the arms or upper extremity veins. The sides of the body where thrombi were found on MRI 
were not equally distributed: most were found on the left side (61\%), $30 \%$ were on the right side, and in $9 \%$ of cases bilateral thrombi were found. Most patients with thrombosis had two or more veins affected ( 25 out of $44,57 \%$ ). Nineteen of the 44 patients $(43 \%)$ had only one occluded vein. The predefined venous segments for the anatomical localization of thrombi are shown in Figure 2 (independently of the side of the DVT). We found that 16 patients $(36 \%)$ with MRI detected thrombosis had occlusions in both the upper and lower leg. The most common origin was the lower leg; 12 of the 44 patients (27\%) had isolated deep calf vein thrombosis, and five $(11 \%)$ presented with isolated superficial vein thrombosis confined to the calf. Abdominal thrombi were rare (3 patients, $7 \%$ ).

We assessed common acquired and genetic risk factors in all participants (Table 3). Overall, $21 \%$ of the patients were aged over 65 years. One in four patients had an active malignancy at the time of PE diagnosis. A similar percentage was found for recent surgery. These risk factors were equally present in PE patients with and without a DVT on MRI. Minor leg injuries were present more often in patients with DVT than in those without DVT, in $11 \%$ and $5 \%$ respectively. One or more immobilization risk factors (i.e., bed-rest of more than 4 days in the preceding 8 weeks, air travel lasting more than 8 hours, travel by car or train for longer than 4 hours) were present in $27 \%$ of patients with DVT, and in $41 \%$ of patients without DVT. Regarding the two genetic risk factors that were assessed in this study, we found a higher prevalence of factor $\mathrm{V}$ Leiden in patients with DVT on MRI (14\%) than in those without DVT (7\%). The prevalences of the prothrombin (G20210A) mutation did not differ much, i.e., $4 \%$ in PE patients without DVT and $2 \%$ in those with DVT.

\section{Discussion}

We performed a total-body MRI scan in 99 patients with $\mathrm{PE}$ and could not detect a peripheral thrombus in more than half of these patients. We had expected to detect thrombi in the majority of cases, and a substantial number in the abdominal or pelvic region where CUS cannot readily detect thrombi. In the following section we present different potential explanations for these surprising findings, some of which we can reject.

\section{Explanation 1: the magnetic resonance direct thrombus imaging technique is not sensitive enough to detect all deep vein thromboses}

Two earlier studies have showed that the sensitivity and specificity of the MRDTI technique are superb for acute DVT of the leg, being between $95-98 \%$ and $96-100 \%$, respectively. ${ }^{11,28}$ The first study compared MRDTI with a reference standard of venography and CUS or venography alone $;^{28}$ the second study used CUS as the gold standard for imaging of DVT. ${ }^{11}$ Our study was not performed to calculate sensitivity and specificity estimates of the MRDTI scanning technique. We did not, therefore, perform ultra-
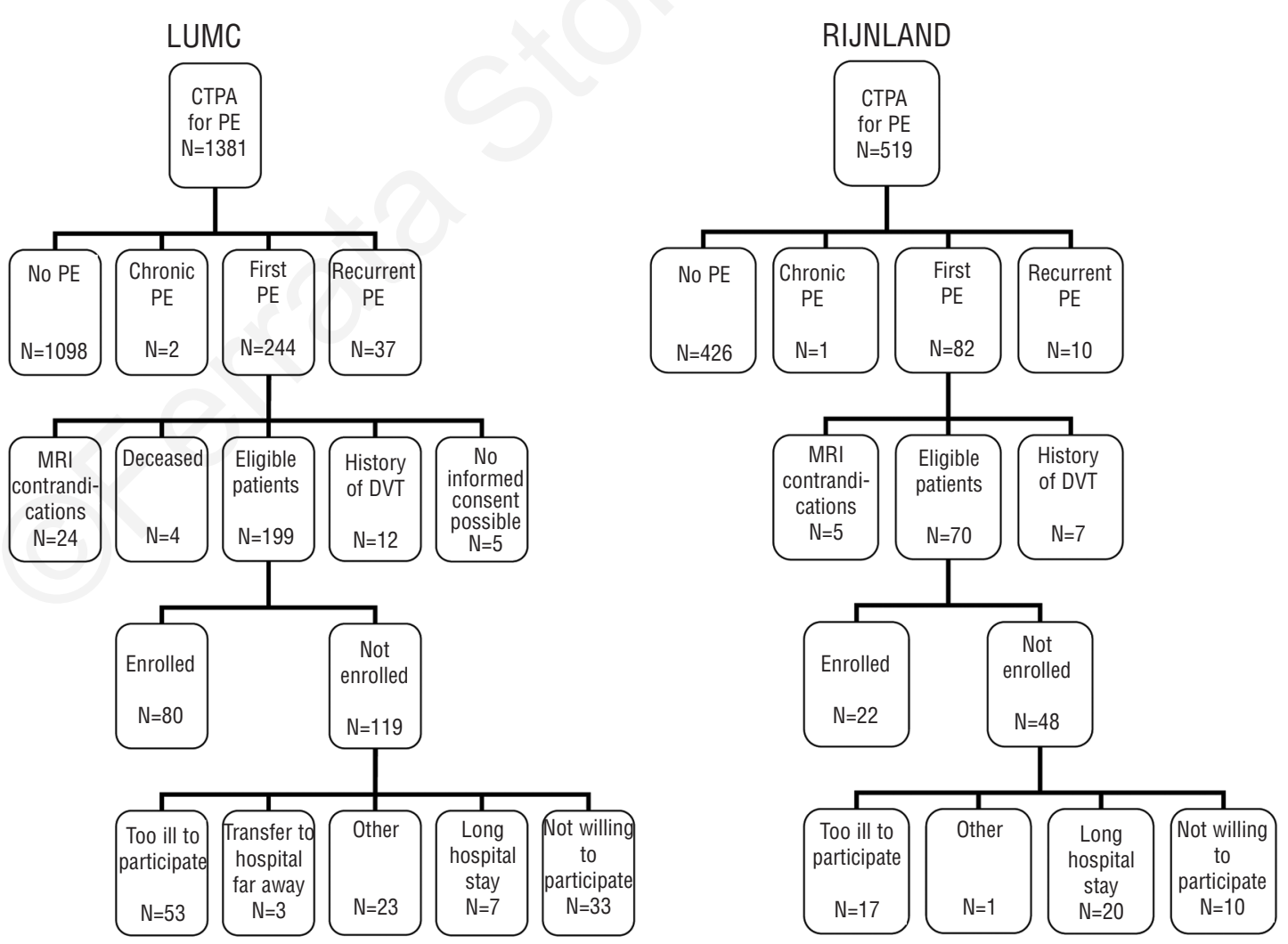

Figure 1. Study patients. Flowchart of patients with pulmonary embolism from the Rijnland Hospital and the Leiden University Medical Center (LUMC). 
sound examinations on all patients by protocol. However, in the 13 patients who did undergo CUS as part of their clinical diagnostic work-up, ten were found by this technique to have DVT. The MRI showed a venous thrombus in nine of these ten patients (i.e., a sensitivity of $90 \%$ ). The DVT that was missed was a (deep) calf vein thrombosis of the left leg. However, even if we accept that not all DVT were detected by MRDTI scanning, this explanation is unlikely to account for $56 \%$ of missing thrombi.

\section{Explanation 2: Pulmonary emboli without deep-vein thrombosis detected by magnetic resonance imaging are due to "therapeutic embolization"}

This explanation for the lack of identification of DVT on MRI in patients with PE is that a thrombus had been present but was completely dislodged to the pulmonary arteries at the time of scanning. This theory seems unlikely as an explanation for all of the missing DVT, as it is not supported by data from post-mortem studies. These studies showed that only part of the clot (usually the free-floating tail) breaks off and embolizes. ${ }^{29-31}$ Furthermore, if this theory were to be true, one would expect that a longer time between the diagnosis of PE and MRI scanning would be related to a lower chance of finding a thrombus. We performed a logistic regression analysis to address this issue, using the presence or absence of DVT as an outcome and the number of days between PE diagnosis and MRI scan as a continuous covariable. Our data showed no association between identification of a DVT and the number of days between diagnosis and MRI, with an odds ratio of 0.99 (95\% CI 0.81-1.21).

\section{Explanation 3: Pulmonary embolism has a cardiac origin}

Case reports describing PE due to right atrial thrombi in patients with atrial fibrillation led us to consider this as an
Table 1. Baseline characteristics of all PE patients.

\begin{tabular}{lc} 
PE patients & $\mathbf{N}=99$ \\
Age (median, range in years) & $54(18-84)$ \\
Sex & \\
Male & $60(61 \%)$ \\
Female & $39(39 \%)$ \\
\hline Body mass index (median, range in $\left.\mathrm{kg} / \mathrm{m}^{2}\right)$ & $26(16-39)$ \\
Hospital of origin & \\
Rijnland Hospital & $22(22 \%)$ \\
Leiden University Medical Center & $77(78 \%)$ \\
\hline In-hospital patients at time of diagnosis & $20(20 \%)$ \\
Duration of complaints (median, range in days) & $2(0-105)$ \\
\hline Clinical ultrasound performed & $13(13 \%)$ \\
DVT on ultrasound & $10(77 \%)$ \\
No DVT on ultrasound & $3(23 \%)$ \\
\hline
\end{tabular}

Table 2. Localization of thrombi in PE patients with a DVT on MRI.

\begin{tabular}{lc} 
PE patients & $\mathbf{N}=99$ \\
No thrombus on MRI & $55(56 \%)$ \\
Thrombus present on MRI & $44(44 \%)$ \\
Right leg DVT & $13(30 \%)$ \\
Left leg DVT & $27(61 \%)$ \\
Bilateral DVT & $4(9 \%)$ \\
1 vein & $19(43 \%)$ \\
2 veins & $12(27 \%)$ \\
3 veins & $3(7 \%)$ \\
4 veins & $4(9 \%)$ \\
5 veins & $4(9 \%)$ \\
6 or more veins & $2(5 \%)$ \\
\hline
\end{tabular}

1. $\mathrm{N}=3$

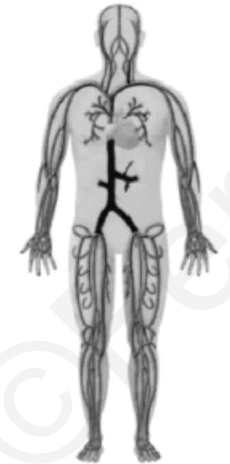

2.

$\mathrm{N}=6$ deep veins $\mathrm{N}=1$ superficial veins

4.

$\mathrm{N}=1$

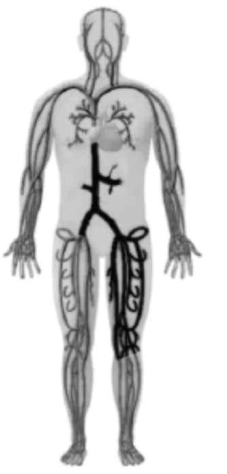

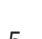

5.

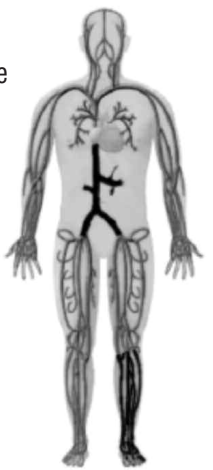

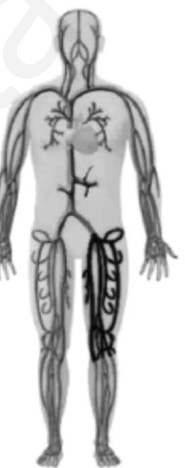

6. $\mathrm{N}=16$

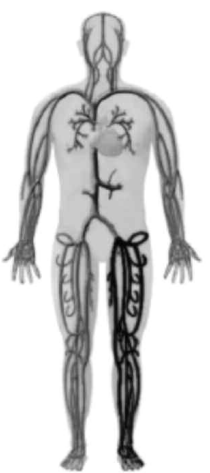

3. veins
$\mathrm{N}=12$ deep veins $\mathrm{N}=5$ superficial

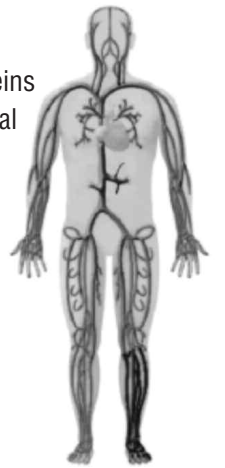

Figure 2. Anatomical distribution of thrombi on MRI. Division into seven venous segments with thrombosis in all patients with pulmonary embolism, with a deep-vein thrombosis on magnetic resonance images $(\mathrm{N}=44)$. 
alternative origin of $\mathrm{PE} .^{32,33}$ In a recent case-control study based on ICD discharge codes in the Danish National Patient Registry, heart disease was shown to be a risk factor for PE without concomitant DVT, with an over 40-fold increased risk in the first 3 months after myocardial infarction or heart failure. ${ }^{34}$ For DVT with or without PE, this risk was increased 10 - to 20 -fold. The risk was more pronounced for right-sided heart disease, suggesting a direct relation with the thrombus in the pulmonary arteries. Two previous studies also showed that patients with atrial fibrillation, mitral valve stenosis or dilated cardiomyopathy are at increased risk of developing isolated PE. The authors concluded that heart diseases could be a source of $\mathrm{PE}$, due to the development of right-sided cardiac throm$\mathrm{bi}^{35,36}$ In our study, in the group of PE patients without DVT on MRI, there were no patients with documented heart failure. Two out of 55 patients had atrial fibrillation (4\%). The absence of heart failure and the low proportion of atrial fibrillation make it unlikely that cardiac thrombi could explain the absence of DVT in over half of the PE patients.

\section{Explanation 4: Pulmonary embolism originates from local thrombus formation in the lungs}

From an alternative etiological point of view, PE may be a local phenomenon, starting in the pulmonary arteries. It has been hypothesized previously that central PE could be caused by DVT while peripheral, smaller PE could be caused by local inflammatory reactions in the pulmonary arteries. $^{31}$ The idea of $\mathrm{PE}$ due to local inflammatory processes was described in two studies performed in trauma patients. ${ }^{31,37}$ Autonomic dysfunction is one of the consequences of trauma and may lead to adrenergia and inflammation that together promote coagulation. Hypoxia may also stimulate the endothelium. ${ }^{31,37}$ Yun et al. concluded that embolic disorders such as PE may represent asynchronous systemic phenomena rather than a clot migration process, when adrenergia and inflammation are present. ${ }^{38}$ In addition, chronic obstructive pulmonary disease and asthma may cause local clots, as persistent low-level systemic inflammation is present in these patients. ${ }^{22,24}$ Data from a nested case-control study showed a 3.6-fold increased risk of PE in patients with mild chronic obstructive pulmonary disease, and a 7.5-fold increased risk of PE among those with severe chronic obstructive pulmonary disease. No increased risk was found for DVT. ${ }^{22}$ We recently found that pneumonia increased the risk of $\mathrm{PE}$ in the MEGA study, with an odds ratio of 8.1 (95\% CI $6.2-$ 10.6). For DVT alone, the odds ratio was 3.0 (95\% CI 2.24.0). ${ }^{23}$

Inflammation affects coagulation through different mechanisms: via tissue factor-mediated thrombin generation, and via the impairment of anticoagulant pathways, the protein $\mathrm{C}$ system in particular. ${ }^{39}$ In asthma patients impaired fibrinolysis has been described. Protease-activated receptors have been pointed out as the molecular link between coagulation and allergic inflammation in asthma. ${ }^{24}$ In addition, coagulation can be further enhanced by the complement system, which is activated during inflammation. One might speculate that local inflammation of the vessel wall leads to thrombus formation.

Another argument that supports the possibility of local $\mathrm{PE}$ is that of recurrent events. If DVT and PE are manifestations of the same disease, the anatomical location of recurrence would be expected to be independent of the
Table 3. Acquired and genetic risk factors in PE patients presenting with or without a thrombus on MRI.

\begin{tabular}{|c|c|c|}
\hline Risk factors & $\begin{array}{c}\text { Thrombus present } \\
\text { on MRI } \\
N=44 \\
\text { n (\%; } 95 \% \text { Cl) }\end{array}$ & $\begin{array}{l}\text { No thrombus } \\
\text { on MRI } \\
N=55 \\
\text { n (\%; } 95 \% \text { Cl) }\end{array}$ \\
\hline Age $>65$ years & $9(20 ; 11-35 \%)$ & $12(22 ; 13-34 \%)$ \\
\hline $\begin{array}{l}\text { Malignancy } \\
\text { History } \\
\text { Active }\end{array}$ & $\begin{array}{c}4(9 ; 4-21 \%) \\
10(23 ; 13-37 \%)\end{array}$ & $\begin{array}{c}6(11 ; 5-22 \%) \\
13(24 ; 14-36 \%)\end{array}$ \\
\hline Surgery in past 8 weeks & $9(20 ; 11-35 \%)$ & $13(24 ; 14-36 \%)$ \\
\hline Minor leg injuries in past 4 weeks & $5(11 ; 5-24 \%)$ & $3(5 ; 2-15 \%)$ \\
\hline Positive family history of VTE & $11(25 ; 15-39 \%)$ & $10(18 ; 10-30 \%)$ \\
\hline Bedrest of $>4$ days in past 8 weeks & $7(16 ; 8-29 \%)$ & $15(27 ; 17-40 \%)$ \\
\hline Air travel of $>8$ h in past 8 weeks & $3(7 ; 2-18 \%)$ & $3(5 ; 2-15 \%)$ \\
\hline $\begin{array}{l}\text { Travel of }>4 \mathrm{~h} \text { by car/train } \\
\text { in the past } 8 \text { weeks }\end{array}$ & $4(9 ; 4-21 \%)$ & $9(16 ; 9-28 \%)$ \\
\hline Any immobilization factor & $12(27 ; 16-42 \%)$ & $22(40 ; 28-53 \%)$ \\
\hline Women using contraceptive pill & $4 / 17(24 ; 10-47 \%)$ & $7 / 22(32 ; 16-53 \%)$ \\
\hline Genetic risk factors & $N=44$ & $N=54$ \\
\hline $\begin{array}{l}\text { Factor V Leiden mutation } \\
\text { Heterozygous } \\
\text { Homozygous }\end{array}$ & $\begin{array}{c}6(14 ; 6-27 \%) \\
-\end{array}$ & $\begin{array}{l}3(6 ; 2-15 \%) \\
1(2 ; 0-10 \%)\end{array}$ \\
\hline $\begin{array}{l}\text { Prothrombin mutation } \\
\text { Heterozygous } \\
\text { Homozygous }\end{array}$ & $\begin{array}{c}1(2 ; 0-12 \%) \\
-\end{array}$ & $2(4 ; 1-13 \%)$ \\
\hline
\end{tabular}

VTE: venous thromboembolism.

location of the first event. However, PE patients are more likely to develop another PE when a recurrence occurs, while patients with DVT have DVT as the recurrent event more often than expected..$^{40}$ In addition, patients with isolated PE are less likely to experience a recurrent event than DVT patients, confirming that isolated PE may be a different entity. ${ }^{41}$

In this study, calf veins were frequently affected in patients with PE (12 patients with thrombi in deep veins and 5 with thrombi in superficial calf veins). Although similar findings have been published previously, ${ }^{5,42-44}$ others have stated that distal thrombi are not relevant as a starting point for PE. ${ }^{45,46}$ Of note, from our data we cannot determine whether a more proximal thrombus had also been present in patients with calf vein DVT and embolized to the lungs, nor can we estimate the risk of embolization of calf vein thromboses. We found thrombi in the abdominal vein segment in only three out of 44 patients $(7 \%)$.

We compared our findings to those of other studies that assessed thrombi above the level of the inguinal ligament, in which imaging of DVT was performed either with CT venography or with magnetic resonance angiography and found inconsistent results. Nchimi and colleagues imaged PE by CT pulmonary angiography followed several minutes later by CT venography for assessment of DVT. The study population had a mean age of 61 years (range 19100 years). Out of $272 \mathrm{PE}$ patients, 200 were found to have concomitant DVT (74\%). Thrombi above the level of the inguinal ligament were found in $10 \%$ of patients with PE, similar to our findings. ${ }^{9}$ In addition, isolated below-knee thrombi were found in more than half of PE patients, and 
the authors concluded that CT venography should be performed including the calves down to the level of the ankle. Velmahos et al. performed combined CT pulmonary angiography and CT venography in 46 trauma patients with PE. The mean age of this series of patients was 56 years. Only seven out of the $46(15 \%)$ patients had a concomitant DVT, of which one was in the abdominal district. $^{31}$

Stern et al. assessed DVT using magnetic resonance angiography in $24 \mathrm{PE}$ patients with negative CUS findings (mean age 49 years; range 18-83). Of these patients seven were found to have pelvic DVT (29\%) and 17 had no concomitant DVT. However, these results cannot be compared directly to our findings due to different study methods, as we did not select patients with a negative CUS, but only with PE proven by CT pulmonary angiography ${ }^{47}$

Of the risk factors we analyzed, the most notable finding was that the factor $\mathrm{V}$ Leiden mutation was present more often $(14 \%)$ in PE patients with a concomitant DVT than in patients with $\mathrm{PE}$ alone $(7 \%)$. This confirms previous findings, which showed that regarding the factor $\mathrm{V}$ Leiden prevalence, patients with both PE and DVT resemble DVT patients more than patients with isolated $\mathrm{PE} \cdot{ }^{13}$ As for minor leg injuries, it seems plausible that patients with an injury of the leg are likely to develop DVT locally, part of which could embolize later. ${ }^{19}$ Patients with isolated PE did indeed have a lower prevalence $(5 \%)$ of minor leg injuries than patients with concomitant DVT (11\%). A limitation to this study is that numbers were small for assessment of differences in risk factors between patients with and without DVT. Our results do, therefore, have to be interpreted carefully and need to be replicated in larger studies.

In summary, in this etiological study we aimed to locate the origin of PE and thereby increase the yield of DVT that were otherwise missed on CUS examinations. We found DVT in less than half of all PE patients using a total-body MRI technique, and proposed several hypotheses to explain the absence of DVT in the majority of patients. Limited sensitivity of MRDTI, complete embolization of the DVT, or a clot of cardiac origin could not sufficiently explain the absence of thrombi. We, therefore, postulate that in some cases PE may arise de novo in the lungs, which could be a new explanation for the origin of $\mathrm{PE}$ without concomitant DVT.

\section{Acknowledgments}

We acknowledge all patients who participated in the study.

We thank the clinicians from the Rijnland hospital and the LUMC for their cooperation with recruitment of patients into the study.

We thank Guido R. van Haren for technical support with the MRI scanning protocol, Petra J. Noordijk for performing the laboratory measurements for the DNA analyses, Hans A. Vogelaar for developing a PROMISE web database for the study and Anne Bosch for administrative support with the enrollment of patients.

\section{Funding}

This study was supported by Netherlands Heart Foundation grant number 2009B050. The funding organization is a public institution and had no role in the design and conduct of the study; collection, management, analysis, and interpretation of the data; and preparation, review, or approval of the manuscript.

\section{Authorship and Disclosures}

Information on authorship, contributions, and financial \& other disclosures was provided by the authors and is available with the online version of this article at www. haematologica.org.

\section{References}

1. Martinelli I, Cattaneo M, Panzeri D, Mannucci PM. Low prevalence of factor $\mathrm{V}: 0506$ in 41 patients with isolated pulmonary embolism. Thromb Haemost. 1997;77(3):440-3.

2. Yamaki T, Nozaki M, Sakurai H, Takeuchi M, Soejima K, Kono T. Presence of lower limb deep vein thrombosis and prognosis in patients with symptomatic pulmonary embolism: preliminary report. Eur J Vasc Endovasc Surg. 2009;37(2):225-31.

3. Jimenez D, Aujesky D, Diaz G, Monreal M, Otero R, Marti D, et al. Prognostic significance of deep vein thrombosis in patients presenting with acute symptomatic pulmonary embolism. Am J Respir Crit Care Med. 2010;181(9):983-91.

4. Girard P, Sanchez O, Leroyer C, Musset D, Meyer G, Stern JB, et al. Deep venous thrombosis in patients with acute pulmonary embolism: prevalence, risk factors, and clinical significance. Chest. 2005;128(3):1593-600.

5. Kruit WH, de Boer AC, Sing AK, van Roon F. The significance of venography in the management of patients with clinically suspected pulmonary embolism. J Intern Med. 1991;230(4):333-9.

6. Hull RD, Hirsh J, Carter CJ, Jay RM, Dodd PE, Ockelford PA, et al. Pulmonary angiography, ventilation lung scanning, and venography for clinically suspected pul- monary embolism with abnormal perfusion lung scan. Ann Intern Med. 1983;98 (6):891-9.

7. Lensing AW, Prandoni P, Prins MH, Büller HR. Deep-vein thrombosis. Lancet. 1999;353(9151):479-85.

8. White RH, McGahan JP, Daschbach MM Hartling RP. Diagnosis of deep-vein thrombosis using duplex ultrasound. Ann Intern Med. 1989;111(4):297-304.

9. Nchimi A, Ghaye B, Noukoua CT, Dondelinger RF. Incidence and distribution of lower extremity deep venous thrombosis at indirect computed tomography venography in patients suspected of pulmonary embolism. Thromb Haemost. 2007;97(4):566-72.

10. Kelly J, Hunt BJ, Moody A. Magnetic resonance direct thrombus imaging: a novel technique for imaging venous thromboemboli. Thromb Haemost. 2003;89(5):773-82.

11. Westerbeek RE, van Rooden CJ, Tan M, van Gils AP, Kok S, de Bats MJ, et al. Magnetic resonance direct thrombus imaging of the evolution of acute deep vein thrombosis of the leg. J Thromb Haemost. 2008;6(7):108792.

12. de Moerloose P, Reber G, Perrier A Perneger T, Bounameaux H. Prevalence of factor V Leiden and prothrombin G20210A mutations in unselected patients with venous thromboembolism. Br J Haematol. 2000;110(1):125-9

13. van Stralen KJ, Doggen CJM, Bezemer ID Pomp ER, Lisman T, Rosendaal FR.
Mechanisms of the factor $\mathrm{V}$ Leiden paradox. Arterioscler Thromb Vasc Biol. 2008;28(10):1872-7.

14. Manten B, Westendorp RG, Koster $T$, Reitsma PH, Rosendaal FR. Risk factor profiles in patients with different clinical manifestations of venous thromboembolism: a focus on the factor $\mathrm{V}$ Leiden mutation. Thromb Haemost. 1996;76(4):510-3.

15. Bounameaux $H$. Factor V Leiden paradox risk of deep-vein thrombosis but not of pulmonary embolism. Lancet. 2000;356(9225): 182-3.

16. Ordonez AJ, Carreira JM, Alvarez CR, Rodriguez JM, Alvarez MV, Coto E. Comparison of the risk of pulmonary embolism and deep vein thrombosis in the presence of factor $V$ Leiden or prothrombin G20210A. Thromb Haemost. 2000;83(2):352-4.

17. Baglin TP, Brown K, Williamson D, Baker $\mathrm{P}$ Luddington $\mathrm{R}$. Relative risk of pulmonary embolism and deep vein thrombosis in association with the factor V Leiden mutation in a United Kingdom population. Thromb Haemost. 1997;77(6):1219

18. Desmarais S, de Moerloose P, Reber G Minazio P, Perrier A, Bounameaux $\mathrm{H}$ Resistance to activated protein $\mathrm{C}$ in an unselected population of patients with pulmonary embolism. Lancet. 1996;347(9012): 1374-5

19. van Langevelde $K$, Flinterman LE, van Hylckama Vlieg A, Rosendaal FR, Cannegieter SC. Broadening the factor V 
